\title{
Stability of active muscle tissue
}

Dorfmann, Luis, luis.dorfmann@tufts.edu; Paetsch, Chris, Tufts University, United States

\begin{abstract}
In this discussion, the notion of material stability is examined in the context of active muscle tissue modeling, where the nonlinear constitutive law is dependent on both the physiologically-driven muscle contraction and the finite mechanical deformation. First, the governing equations and constitutive laws for a general active-elastic material are linearized about a homogeneous underlying configuration. In order to obtain mathematical restrictions analogous to those found in elastic materials, stability conditions are derived based on the propagation of homogeneous plane waves with real wave speeds, and the generalized acoustic tensor is obtained. Focusing on 2D motions, and considering a simplified, decoupled transversely isotropic energy function, the restriction on the active acoustic tensor is recast in terms of a generally applicable constitutive law, with specific attention paid to the fiber contribution. The implication of the material stability conditions on material parameters, active contraction, and elastic stretch is investigated for prototype material models of muscle tissue.
\end{abstract}

